\title{
Video-based quantification of human movement frequency using pose estimation
}

\author{
Hannah L. Cornman ${ }^{1,2,3}$, Jan Stenum, $\mathrm{PhD}^{1,2}$, and *Ryan T. Roemmich, $\mathrm{PhD}^{1,2}$ \\ ${ }^{1}$ Center for Movement Studies, Kennedy Krieger Institute, Baltimore, MD 21205 \\ ${ }^{2}$ Dept of Physical Medicine and Rehabilitation, Johns Hopkins University School of Medicine, \\ Baltimore, MD 21205 \\ ${ }^{3}$ University of Maryland School of Medicine, Baltimore, MD 21201 \\ *corresponding author
}

\section{Contact information:}

G-04 Kennedy Krieger Institute

707 N Broadway

Baltimore, MD 21205

rroemmi1@jhmi.edu

Abstract word count: 249 Text word count: 3799

Number of figures: 6

Keywords: Pose estimation, movement frequency, repetitive movements, computer vision, artificial intelligence

Running head: Measurement of movement frequency using pose estimation 


\section{ABSTRACT}

Assessment of repetitive movements (e.g., finger tapping) is a hallmark of motor

3 examinations in several neurologic populations. These assessments are traditionally performed

4 by a human rater via visual inspection; however, advances in computer vision offer potential for

5 remote, quantitative assessment using simple video recordings. Here, we evaluated a pose

6 estimation approach for measurement of human movement frequency from smartphone videos.

7 Ten healthy young participants provided videos of themselves performing five repetitive

8 movement tasks (finger tapping, hand open/close, hand pronation/supination, toe tapping, leg

9 agility) at four target frequencies (1-4 Hz). We assessed the ability of a workflow that

10 incorporated OpenPose (a freely available whole-body pose estimation algorithm) to estimate

11 movement frequencies by comparing against manual frame-by-frame (i.e., ground-truth)

12 measurements for all tasks and target frequencies using repeated measures ANOVA, Pearson's

13 correlations, and intraclass correlations. Our workflow produced largely accurate estimates of

14 movement frequencies; only the hand open/close task showed a significant difference in the

15 frequencies estimated by pose estimation and manual measurement (while statistically

16 significant, these differences were small in magnitude). All other tasks and frequencies showed

17 no significant differences between pose estimation and manual measurement. Pose estimation-

18 based detections of individual events (e.g., finger taps, hand closures) showed strong correlations

19 with manual detections for all tasks and frequencies. In summary, our pose estimation-based

20 workflow accurately tracked repetitive movements in healthy adults across a range of tasks and

21 movement frequencies. Future work will test this approach as a fast, low-cost, accessible

22 approach to quantitative assessment of repetitive movements in clinical populations. 
INTRODUCTION

Several neurologic conditions - including Parkinson's disease (PD; 1) and cerebellar disorders (2) - impair the ability to execute repetitive movements. This has led to the inclusion of repetitive movements in many clinical examinations of motor function. For example, the MDS-UPDRS (the clinical standard for motor assessment in PD) contains several items that test repetitive movements across different tasks, including finger tapping, hand opening and closing, hand pronation and supination, toe tapping, and leg agility (3). These assessments provide important insights into disease severity that influence treatment decisions and enable tracking of disease progression over time. neurologist rates the performance of each repetitive movement task from 0 (no impairment) to 4

35 (severe impairment) via visual assessment. Other approaches have used research-grade

36 equipment (e.g., motion capture systems), wearables (4,5), and mobile devices (6-8) to quantify

37 performance of repetitive movements. However, motion capture systems are inaccessible to

38 many clinicians and researchers, and wearable- or smartphone-based approaches typically assess

39 only specific movements (e.g., finger tapping). There is a need for a rapid, low-cost approach

40 capable of providing quantitative measurement of repetitive movements across multiple

41 extremities using equipment that is readily accessible in the home or clinic.

43 capable of tracking human movement with a high degree of accuracy and minimal technological

44 requirement (9-14). These algorithms have shown an impressive ability to track specific features

45 of the human body (e.g., fingers, leg joints) from digital videos recorded by common household 
46 devices (e.g., smartphones, tablets, laptop computers). There is significant potential for these

47 approaches to provide rapid, automated measurement and analysis of repetitive movements from

48 videos recorded in the home or clinic, enabling remote assessment that does not require a patient

49 visit to the clinic or manual assessment from a clinician.

In this study, we used OpenPose (a freely available human pose estimation algorithm

$51(11,15,16)$ to measure the frequencies of repetitive upper and lower extremity movements using

52 smartphone videos. Previous studies have used OpenPose to study human gait (17-21) and

53 quantify tremor severity in persons with PD (22). Other pose estimation algorithms have been

54 used to measure the degree of levodopa-induced dyskinesias (23) and finger tapping frequency in

55 PD that correlated well with clinical ratings $(24,25)$. While emerging evidence suggests

56 significant potential for tracking repetitive movements using video-based pose estimation

57 approaches, here we addressed two important unmet needs: 1) testing across a range of clinically

58 relevant tasks spanning the upper and lower extremities, and 2) assessment of accuracy via

59 validation against ground-truth measurement. Based on our prior work (21), we expected good

60 agreement between the OpenPose estimates of movement frequency and ground-truth

61 measurement. We also share our perspectives regarding limitations, potential problems, and

62 suggestions for successful analysis. All code used for this project is freely available at

63 https://github.com/RyanRoemmich/PoseEstimationApplications.

\section{MATERIALS AND METHODS}

\section{Participants}

66 We recruited ten healthy adults (four male, six female, age (mean \pm standard deviation):

$6728 \pm 4$ years) to participate. All participants provided written informed consent in accordance

68 with the Johns Hopkins University School of Medicine Institutional Review Board. 


\section{Setup:}

\section{Data collection}

Participants provided digital videos of themselves performing five repetitive movement tasks included on the MDS-UPDRS (3): finger tapping, hand opening and closing, hand pronation and supination, toe tapping, and leg agility (i.e., heel tapping). We asked participants to perform each task at four target frequencies $(1 \mathrm{~Hz}, 2 \mathrm{~Hz}, 3 \mathrm{~Hz}$, and $4 \mathrm{~Hz}$ ) using an online metronome for approximately 10 seconds each. Each participant received instructions for filming the videos and we provided example videos of each task to promote uniformity in the recordings. The instructions were as follows:

- Sit in a chair with knees pointing forward and feet flat on the floor (with the exception of hand pronation/supination, where we asked participants to record sagittal views "record from the side" - of themselves performing the movement). - Make sure hand is held to the side when performing hand and finger tasks, not in front of the body.

- The phone should be approximately five feet from the participant and at their eye level when filming.

- Capture the whole body.

- Film with a smartphone camera at standard sampling rate (approximately 30 frames/sec).

- Use an online metronome to pace movement frequency and try to match the metronome as closely as possible.

Video recording: 
- Record approximately 10 seconds of the participant performing each of the following tasks with one hand at 4 different speeds (1-4 Hz).

- Finger tapping: tap index finger to the thumb (opening as big as possible between taps). - Hand opening and closing: fully open and close the hand repeatedly (opening as big as possible between hand closures).

- Hand pronation and supination: alternate pronating and supinating the hand (pronate on one beat, supinate on the next).

- Toe tapping: keep the heel planted and tap the toe to the ground.

- Leg agility: lift the entire foot and strike the ground with heel.

\section{Pose estimation}

Our data analysis workflow is shown in Figure 1. First, we analyzed video recordings in OpenPose using a Python environment to obtain two-dimensional pixel coordinates of OpenPose keypoints. We used both the Hand (26) and BODY_25 (16) keypoint models simultaneously.

The Hand keypoint model tracks 21 keypoints on each hand: one on each tip and all three joints per finger, and one at the base of the hand. The BODY_25 keypoint model tracks keypoints at the nose, neck, midhip, and bilateral eyes, ears, shoulders, elbows, wrists, hips, knees, ankles, heels, big toes, and small toes. The outputs of the OpenPose analyses yielded: 1) JSON files containing frame-by-frame pixel coordinates of each keypoint, and 2) an output video file in which a skeleton figure representing the detected keypoints is overlaid onto the original video recording. We then analyzed the JSON files using custom MATLAB (Mathworks, Natick, MA) software to calculate movement frequency (detailed below).

\section{Calculation of movement frequency}


We performed several steps to calculate movement frequencies from the JSON files.

114 First, we filled gaps in all keypoint trajectories (i.e., frames where OpenPose did not detect all

115 keypoints) using linear interpolation. Second, we filtered keypoint trajectories using a zero-lag

$1164^{\text {th }}$ order low-pass Butterworth filter with a cut-off frequency at $5 \mathrm{~Hz}$. Third, we identified events

117 (finger taps, hand closures, hand pronations/supinations, toe taps, heel taps) using the following

118 criteria:

a) Finger tapping and hand opening and closing tasks: local minima in the distance between index finger and thumb keypoints.

b) Hand pronation and supination: local maxima (thumb up, supination) and minima (thumb down, pronation).

c) Toe tapping: local minima of the big toe keypoint.

d) Leg agility: local minima of the heel keypoint.

125 Finally, we calculated movement frequency from the identified event times.

\section{Manual ground-truth identification of event times}

We obtained a ground-truth benchmark by manually identifying event times through

128 frame-by-frame visual inspection for all videos. We used the same criteria listed above to

129 identify events manually. From these manually-identified event times, we calculated ground-

130 truth movement frequency.

$131 \quad$ Statistical analysis

132 We compared movement frequencies between methods and among target frequencies

133 using a $2 \times 4$ method (pose estimation, manual) $\mathrm{x}$ target frequency $(1,2,3,4 \mathrm{~Hz})$ repeated

134 measures ANOVA. All events identified by the pose estimation and manual methods were

135 included in calculations of movement frequencies. 
For all correlational analyses, we isolated events that had been detected by both the pose

137 estimation and manual identification methods (the overwhelming majority of events were

138 detected by both methods, as described in the Results section below). To do this, we first

139 compared the number of events identified by both methods in each individual video. If the

140 numbers of events were not equal between the two methods (i.e., either the pose estimation

141 approach identified more events than the manual identification or vice versa), we discarded the

142 events from the method with more detected events that had video timestamps ("event times")

143 most dissimilar to any event time detected by the other method until the number of detected

144 events were equivalent in both methods. Most often, this meant that the pose estimation approach

145 detected (or failed to detect) an additional event at the beginning or end of the trial, and these

146 events that were not detected by both methods were then discarded from the correlational

147 analyses. We also observed a few trials where both methods detected the same number of events,

148 but one method had detected an additional event at the beginning of the trial and the other an

149 additional event at the end of the trial; in this case, we discarded both unmatched events. From

150 the matched events, we calculated Pearson correlation coefficients $(r)$ and intra-class correlation

151 coefficients $\left(\mathrm{ICC}_{\mathrm{C}-1}\right.$ and $\left.\mathrm{ICC}_{\mathrm{A}-1}\right)$ to assess correlations $(r)$, consistency $\left(\mathrm{ICC}_{\mathrm{C}-1}\right)$ and agreement

$152\left(\mathrm{ICC}_{\mathrm{A}-1}\right)$ between event time estimates by our pose estimation approach and those identified

153 manually. We used an alpha level of 0.05 for all analyses and performed post-hoc pairwise

154 comparisons with Bonferroni corrections where appropriate.

\section{RESULTS}

$156 \quad$ Finger tapping

157 Repeated measures ANOVA revealed a significant main effect of target frequency

$158(\mathrm{~F}(3,27)=416.31, \mathrm{p}<0.001)$ but no significant main effect of method $(\mathrm{F}(1,9)=1.43, \mathrm{p}=0.26)$ or 
target frequency $x$ method interaction $(\mathrm{F}(3,27)=0.97, \mathrm{p}=0.42)$ on frequency of finger tapping

160 (Figure 2A). As expected, post-hoc analyses revealed significant differences in movement

161 frequency between each pair of target frequencies (all $\mathrm{p}<0.001$ ). This confirmed that participants

162 changed their tapping frequency in accordance with the metronome-paced target frequencies, and

163 these changes were similar in the pose estimation and ground-truth measurements.

Across all trials, our pose estimation approach identified 993 finger taps and manual identification detected 1002 finger taps across all participants and trials; of these, there were 986

167 estimation and manual detections of each finger tap (i.e., time of manually-detected finger tap

168 minus time of pose estimation-detected finger tap) for all taps detected by both methods are

169 shown in Figure 2B. Figure 2C shows associations between manually-detected finger tap times

170 and pose estimation-detected finger tap times for target frequencies of $1 \mathrm{~Hz}(r=1.00, \mathrm{p}<0.001$;

$\left.171 \quad \mathrm{ICC}_{\mathrm{C}-1}=1.00, \mathrm{p}<0.001 ; \mathrm{ICC}_{\mathrm{A}-1}=1.00, \mathrm{p}<0.001\right), 2 \mathrm{~Hz}\left(r=1.00, \mathrm{p}<0.001 ; \mathrm{ICC}_{\mathrm{C}-1}=1.00, \mathrm{p}<0.001 ;\right.$

$\left.\operatorname{ICC}_{\mathrm{A}-1}=1.00, \mathrm{p}<0.001\right), 3 \mathrm{~Hz}\left(r=1.00, \mathrm{p}<0.001 ; \operatorname{ICC}_{\mathrm{C}-1}=1.00, \mathrm{p}<0.001 ; \operatorname{ICC}_{\mathrm{A}-1}=1.00, \mathrm{p}<0.001\right)$,

173 and $4 \mathrm{~Hz}\left(r=1.00, \mathrm{p}<0.001 ; \mathrm{ICC}_{\mathrm{C}-1}=1.00, \mathrm{p}<0.001 ; \mathrm{ICC}_{\mathrm{A}-1}=1.00, \mathrm{p}<0.001\right)$.

\section{$174 \quad$ Hand opening and closing}

Repeated measures ANOVA revealed a significant main effect of target frequency

$176(\mathrm{~F}(3,27)=1032.07, \mathrm{p}<0.001)$, a significant main effect of method $(\mathrm{F}(1,9)=9.08, \mathrm{p}=0.02)$, and a

177 significant target frequency $x$ method interaction $(F(3,27)=4.70, p=0.01)$ on frequency of hand

178 opening and closing (Figure 3A). Post-hoc analyses revealed significant differences in movement

179 frequency between each pair of target frequencies (all $\mathrm{p}<0.001)$. The movement frequencies

180 estimated by the pose estimation approach were significantly slower than the frequencies

181 identified manually at target frequencies of $2 \mathrm{~Hz}, 3 \mathrm{~Hz}$, and $4 \mathrm{~Hz}$ (all p<0.02); however, the 
magnitudes of these differences were very small (mean difference between methods no larger

183

184

185

than $0.03 \mathrm{~Hz}$ for any target frequency).

Across all trials, our pose estimation approach identified 960 hand closures and manual identification detected 973 hand closures across all participants and trials; of these, there were 955 hand closures detected by both methods. Distributions of errors between the pose estimation and manual detections of each hand closure detected by both methods are shown in Figure 3B. Figure 3C shows associations between manually-detected hand closure times and pose estimation-detected hand closure times for target frequencies of $1 \mathrm{~Hz}(r=1.00, \mathrm{p}<0.001$; ICC $\mathrm{C}$ $\left.{ }_{1}=1.00, \mathrm{p}<0.001 ; \mathrm{ICC}_{\mathrm{A}-1}=1.00, \mathrm{p}<0.001\right), 2 \mathrm{~Hz}\left(r=1.00, \mathrm{p}<0.001 ; \mathrm{ICC}_{\mathrm{C}-1}=1.00, \mathrm{p}<0.001 ; \mathrm{ICC}_{\mathrm{A}-}\right.$ $\left.{ }_{1}=1.00, \mathrm{p}<0.001\right), 3 \mathrm{~Hz}\left(r=1.00, \mathrm{p}<0.001 ; \mathrm{ICC}_{\mathrm{C}-1}=1.00, \mathrm{p}<0.001 ; \mathrm{ICC}_{\mathrm{A}-1}=1.00, \mathrm{p}<0.001\right)$, and 4 $\mathrm{Hz}\left(r=1.00, \mathrm{p}<0.001 ; \operatorname{ICC}_{\mathrm{C}-1}=1.00, \mathrm{p}<0.001 ; \operatorname{ICC}_{\mathrm{A}-1}=1.00, \mathrm{p}<0.001\right)$.

\section{Hand pronation and supination}

Repeated measures ANOVA revealed a significant main effect of target frequency $(\mathrm{F}(3,27)=656.54, \mathrm{p}<0.001)$, but no significant main effect of method $(\mathrm{F}(1,9)=0.33, \mathrm{p}=0.58)$ or target frequency $\mathrm{x}$ method interaction $(\mathrm{F}(3,27)=2.15, \mathrm{p}=0.12)$ on frequency of alternating hand pronation and supination (Figure 4A). Post-hoc analyses revealed significant differences in movement frequency between each pair of target frequencies (all p<0.001).

Across all trials, our pose estimation approach identified 974 hand pronation or supination events and manual identification detected 991 hand pronation or supination events across all participants and trials; of these, there were 958 common hand pronation or supination events detected by both methods. Distributions of errors between the pose estimation and manual detections of each hand pronation or supination event detected by both methods are shown in Figure 4B. Figure 4C shows associations between manually-detected hand pronation or 
supination event times and pose estimation-detected hand pronation or supination event times for

206

207

208

209

210

211

212

213

214

215

216

217

218

219

220

221

222

223

$\left.2241=1.00, \mathrm{p}<0.001 ; \mathrm{ICC}_{\mathrm{A}-1}=1.00, \mathrm{p}<0.001\right)$.

225 Leg agility

226

227

$\left.\mathrm{ICC}_{\mathrm{A}-1}=1.00, \mathrm{p}<0.001\right)$.

\section{Toe tapping} each pair of target frequencies (all $\mathrm{p}<0.001)$.

target frequencies of $1 \mathrm{~Hz}\left(r=1.00, \mathrm{p}<0.001 ; \mathrm{ICC}_{\mathrm{C}-1}=1.00, \mathrm{p}<0.001 ; \mathrm{ICC}_{\mathrm{A}-1}=1.00, \mathrm{p}<0.001\right), 2 \mathrm{~Hz}$ $\left(r=1.00, \mathrm{p}<0.001 ; \mathrm{ICC}_{\mathrm{C}-1}=1.00, \mathrm{p}<0.001 ; \mathrm{ICC}_{\mathrm{A}-1}=1.00, \mathrm{p}<0.001\right), 3 \mathrm{~Hz}\left(r=1.00, \mathrm{p}<0.001 ; \mathrm{ICC}_{\mathrm{C}-}\right.$

$\left.{ }_{1}=1.00, \mathrm{p}<0.001 ; \mathrm{ICC}_{\mathrm{A}-1}=1.00, \mathrm{p}<0.001\right)$, and $4 \mathrm{~Hz}\left(r=1.00, \mathrm{p}<0.001 ; \mathrm{ICC}_{\mathrm{C}-1}=1.00, \mathrm{p}<0.001\right.$;

Repeated measures ANOVA revealed a significant main effect of target frequency $(F(3,27)=794.24, p<0.001)$, but no significant main effect of method $(F(1,9)=4.86, p=0.06)$ or target frequency $\mathrm{x}$ method interaction $(\mathrm{F}(3,27)=2.18, \mathrm{p}=0.11)$ on frequency of toe tapping

(Figure 5A). Post-hoc analyses revealed significant differences in movement frequency between

Across all trials, our pose estimation approach identified 989 toe taps and manual identification detected 1003 toe taps across all participants and trials; of these, there were 969 common toe taps detected by both methods. Distributions of errors between the pose estimation and manual detections of each toe tap detected by both methods are shown in Figure 5B. Figure 5C shows associations between manually-detected toe tap times and pose estimation-detected toe tap times for target frequencies of $1 \mathrm{~Hz}\left(r=1.00, \mathrm{p}<0.001 ; \mathrm{ICC}_{\mathrm{C}-1}=1.00, \mathrm{p}<0.001 ; \mathrm{ICC}_{\mathrm{A}-1}=1.00\right.$, $\mathrm{p}<0.001), 2 \mathrm{~Hz}\left(r=1.00, \mathrm{p}<0.001 ; \mathrm{ICC}_{\mathrm{C}-1}=1.00, \mathrm{p}<0.001 ; \mathrm{ICC}_{\mathrm{A}-1}=1.00, \mathrm{p}<0.001\right), 3 \mathrm{~Hz}(r=1.00$, $\left.\mathrm{p}<0.001 ; \mathrm{ICC}_{\mathrm{C}-1}=1.00, \mathrm{p}<0.001 ; \mathrm{ICC}_{\mathrm{A}-1}=1.00, \mathrm{p}<0.001\right)$, and $4 \mathrm{~Hz}\left(r=1.00, \mathrm{p}<0.001 ; \mathrm{ICC}_{\mathrm{C}-}\right.$

Repeated measures ANOVA revealed a significant main effect of target frequency $(\mathrm{F}(3,27)=2005.84, \mathrm{p}<0.001)$, but no significant main effect of method $(\mathrm{F}(1,9)=2.66, \mathrm{p}=0.14)$ or 
target frequency $x$ method interaction $(F(3,27)=1.69, \mathrm{p}=0.19)$ on frequency of heel tapping

229

230

231

232

233

234

235

236

237

238

239

240

241

242

243

244

245

246

247

248

249

250

(Figure 6A). Post-hoc analyses revealed significant differences in movement frequency between each pair of target frequencies (all $\mathrm{p}<0.001)$.

Across all trials, our pose estimation approach identified 1009 heel taps and manual identification detected 1001 heel taps across all participants and trials; of these, there were 996 common heel taps detected by both methods. Distributions of errors between the pose estimation and manual detections of each heel tap detected by both methods are shown in Figure 6B. Figure 6C shows associations between manually-detected heel tap times and pose estimation-detected heel tap times for target frequencies of $1 \mathrm{~Hz}\left(r=1.00, \mathrm{p}<0.001 ; \mathrm{ICC}_{\mathrm{C}-1}=1.00, \mathrm{p}<0.001\right.$; ICC $_{\mathrm{A}}$ $\left.{ }_{1}=1.00, \mathrm{p}<0.001\right), 2 \mathrm{~Hz}\left(r=1.00, \mathrm{p}<0.001 ; \operatorname{ICC}_{\mathrm{C}-1}=1.00, \mathrm{p}<0.001 ; \operatorname{ICC}_{\mathrm{A}-1}=1.00, \mathrm{p}<0.001\right), 3 \mathrm{~Hz}$ $\left(r=1.00, \mathrm{p}<0.001 ; \operatorname{ICC}_{\mathrm{C}-1}=1.00, \mathrm{p}<0.001 ; \mathrm{ICC}_{\mathrm{A}-1}=1.00, \mathrm{p}<0.001\right)$, and $4 \mathrm{~Hz}(r=1.00, \mathrm{p}<0.001 ;$ $\left.\mathrm{ICC}_{\mathrm{C}-1}=1.00, \mathrm{p}<0.001 ; \mathrm{ICC}_{\mathrm{A}-1}=1.00, \mathrm{p}<0.001\right)$.

\section{DISCUSSION}

In this study, we demonstrated that pose estimation can be used to measure movement frequency across upper and lower extremity tasks in healthy adults. We used the pretrained human network included in the OpenPose demo to track the movements of healthy adults as they performed five different repetitive movement tasks (finger tapping, hand opening and closing, hand pronation and supination, toe tapping, and leg agility) at target frequencies spanning 1-4 Hz. We observed that our analysis workflow accurately detected movement events (e.g., finger taps, hand closures) and estimated movement frequencies that closely agreed with ground-truth measurements derived from manual inspection across all tasks and frequencies. Our results demonstrate that pose estimation can be used as a fast, inexpensive, video-based approach for quantitatively measuring human movement frequencies across upper and lower extremity tasks. 
Interest in pose estimation has increased rapidly in the machine learning and

252 neuroscience communities (9-14,27); however, clinical applications in humans are limited

$253(18,22-24,28)$. The pros and cons of using currently available pose estimation algorithms for

254 measurement of human movement have been discussed at length (29), and we will discuss our

255 own impressions and suggestions below. Despite current limitations, this study and our prior

256 work (21) have shown promising results when comparing pose estimation outputs to ground-

257 truth measurements of clinically relevant movement parameters. We anticipate that the accuracy

258 of human pose estimation algorithms will continue to improve, and we offer that this emerging

259 technology has significant potential for versatile, quantitative, remote assessment of human

260 motor function.

261 The repetitive movement tasks included in this study were selected because they are used

262 in the MDS-UPDRS (3) to assess bradykinesia of the upper and lower extremities in persons

263 with PD. This study is intended to be a preliminary step toward development of a quantitative,

264 comprehensive, video-based approach for assessment of bradykinesia of the upper and lower

265 extremities in persons with PD, and future work will be needed to assess the accuracy of

266 movement frequency estimation in patient populations. Moreover, applications for PD in

267 particular will need to expand upon our workflow to incorporate assessments of movement

268 amplitude in addition to frequency.

269 To calculate movement frequencies, we detected event times manually (ground-truth) and

270 using OpenPose keypoint trajectories. Across tasks, we tended to observe event time errors (i.e.,

271 differences between manual and pose estimation detections) of 1-2 video frames on average.

272 This suggests a small systematic bias in event detection between the two methods. However, we

273 note that movement frequency can be estimated accurately since a phase shift (bias in event 
274 times) will preserve the true frequency despite differences in when the event times are detected.

275 This is illustrated from our results demonstrating that OpenPose estimated movement

276 frequencies well across a repertoire of motor tasks.

277 Other novel techniques that rely on wearables and mobile devices have been proposed to

278 quantify performance of repetitive movement (4-8). While we show here that movement

279 frequencies of tasks included in Part III of the MDS-UPDRS can be accurately obtained using

280 video-based pose estimation in healthy adults, we did not test this approach against other device-

281 based quantification methods. Indeed, other measurement devices may offer advantages that are

282 difficult to quantify with our method. For example, wearable inertial measurement units (IMUs)

283 may be more appropriate for free-living monitoring over longer durations. It is possible that

284 different approaches could be integrated into a more comprehensive approach using different

285 types of sensors to track various movement characteristics in PD. For quantitative information on

286 repetitive movement in PD for clinical use, we propose that pose estimation may be favorable

287 due to its ease of use, computational simplicity, and demonstrated accuracy.

We also found that our approach was quite versatile, as successful analysis of videos was

289 achieved by variable methods of self-recording by participants, in variable environments, and

290 with variable recording devices. Some participants placed a phone on a stable surface at the

291 desired distance and height (as described in methods) and self-recorded without the assistance of

292 another person. Others asked a family member or friend to record them according to these same

293 guidelines, and both methods produced successful analyses. Additionally, participants recorded

294 the videos in various locations and used their personal smartphones (the model varied among

295 participants), and successful analyses were achieved for all participants. This underscores a 
296 significant advantage that this technique could have in clinical practice: all persons with a

297 smartphone or tablet can likely record videos that could be successfully analyzed.

299 below. First, we chose to film the entire body and use both the OpenPose BODY_25 and Hand

300 keypoint models because, in preliminary trials, this produced better hand keypoint detection than

301 simply filming the hand and using the Hand keypoint model alone. Second, filming hand

302 pronation/supination from the frontal plane (i.e., participant directly facing the camera with arms

303 extended directly toward the camera) caused difficulty with consistent hand keypoint detection

304 during pilot data collection. To address this issue, we asked participants to film all hand

305 pronation/supination tasks in the sagittal plane ("from the side"). We found that this significantly

306 improved hand keypoint tracking. A third observation was that, during finger tapping and hand

307 opening and closing tasks, we achieved better tracking when the participant held the hand to the

308 side of the body rather than extended in front of the torso (due to less overlap of the body

309 keypoint tags with the hand keypoints). Finally, we observed that some postures (e.g., sitting

310 with legs crossed) occasionally caused difficulty with BODY_25 keypoint tracking; therefore,

311 we instructed participants to sit with "knees pointing forward and feet flat on the floor".

\section{CONCLUSIONS}

313 In this study, we used a pose estimation approach for fast, quantitative measurement of

314 movement frequency during several upper and lower extremity tasks that are commonly included

315 on neurological examinations. We found that this approach provided largely accurate

316 identifications of event times and estimations of movement frequencies when compared to

317 ground-truth measurements. Future work will aim to establish similar validity in clinical

318 populations. 
bioRxiv preprint doi: https://doi.org/10.1101/2021.02.01.429161; this version posted February 2, 2021. The copyright holder for this preprint (which was not certified by peer review) is the author/funder. All rights reserved. No reuse allowed without permission.

\section{ACKNOWLEDGMENTS}

320 We acknowledge funding from the Association of Academic Physiatrists Rehabilitation

321 Research Experience for Medical Students Fellowship to HLC and NIH grant R21AG059184 to 322 RTR. 


\section{REFERENCES}

324 1. Fahn S. Description of Parkinson's disease as a clinical syndrome. In: Annals of the New $325 \quad$ York Academy of Sciences. 2003.

326 2. Diener H -C, Dichgans J. Pathophysiology of cerebellar ataxia. Movement Disorders. 1992.

3. Goetz CG, Tilley BC, Shaftman SR, Stebbins GT, Fahn S, Martinez-Martin P, et al. Movement Disorder Society-Sponsored Revision of the Unified Parkinson's Disease Rating Scale (MDS-UPDRS): Scale presentation and clinimetric testing results. Mov Disord. 2008;

4. di Biase L, Summa S, Tosi J, Taffoni F, Marano M, Rizzo AC, et al. Quantitative analysis of bradykinesia and rigidity in Parkinson's disease. Front Neurol. 2018; system for quantification of bradykinesia based on wearable inertial sensors. Sensors (Switzerland). 2019;

337 6. Lalvay L, Lara M, Mora A, Alarcón F, Fraga M, Pancorbo J, et al. Quantitative Measurement of Akinesia in Parkinson’s Disease. Mov Disord Clin Pract. 2017;

339 7. Chen O, Lipsmeier F, Phan H, Prince J, Taylor K, Gossens C, et al. Building a Machine340 learning Framework to Remotely Assess Parkinson's Disease Using Smartphones. IEEE $341 \quad$ Trans Biomed Eng. 2020;

342 8. Zhan A, Mohan S, Tarolli C, Schneider RB, Adams JL, Sharma S, et al. Using smartphones and machine learning to quantify Parkinson disease severity the mobile Parkinson disease score. JAMA Neurol. 2018;

345 9. Mathis A, Mamidanna P, Cury KM, Abe T, Murthy VN, Mathis MW, et al. DeepLabCut: 
markerless pose estimation of user-defined body parts with deep learning. Nat Neurosci. 2018

348 10. Insafutdinov E, Pishchulin L, Andres B, Andriluka M, Schiele B. Deepercut: A deeper, stronger, and faster multi-person pose estimation model. In: Lecture Notes in Computer Science (including subseries Lecture Notes in Artificial Intelligence and Lecture Notes in Bioinformatics). 2016.

352 11. Cao Z, Simon T, Wei SE, Sheikh Y. Realtime multi-person 2D pose estimation using part affinity fields. In: Proceedings - 30th IEEE Conference on Computer Vision and Pattern Recognition, CVPR 2017. 2017.

12. Insafutdinov E, Andriluka M, Pishchulin L, Tang S, Levinkov E, Andres B, et al.

13. Pishchulin L, Insafutdinov E, Tang S, Andres B, Andriluka M, Gehler P, et al. DeepCut:

14. Toshev A, Szegedy C. DeepPose: Human pose estimation via deep neural networks. In: Recognition. 2014. Vision. 2019.

367 16. Cao Z, Hidalgo Martinez G, Simon T, Wei S-E, Sheikh YA. OpenPose: Realtime Multi- 
Intell. 2019;

370 17. Chambers C, Kong G, Wei K, Kording K. Pose estimates from online videos show that side-by-side walkers synchronize movement under naturalistic conditions. PLoS One. 2019;

373 18. Sato K, Nagashima Y, Mano T, Iwata A, Toda T. Quantifying normal and parkinsonian gait features from home movies: Practical application of a deep learning-based 2D pose estimator. PLoS One. 2019;

19. Viswakumar A, Rajagopalan V, Ray T, Parimi C. Human Gait Analysis Using OpenPose. In: Proceedings of the IEEE International Conference Image Information Processing.

379 20. Zago M, Luzzago M, Marangoni T, De Cecco M, Tarabini M, Galli M. 3D Tracking of Human Motion Using Visual Skeletonization and Stereoscopic Vision. Front Bioeng Biotechnol. 2020;

21. Stenum J, Rossi C, Roemmich RT. Two-dimensional video-based analysis of human gait using pose estimation. bioRxiv [Internet]. 2020; Available from: https://www.biorxiv.org/content/10.1101/2020.07.24.218776v1

22. Pang Y, Christenson J, Jiang F, Lei T, Rhoades R, Kern D, et al. Automatic detection and quantification of hand movements toward development of an objective assessment of tremor and bradykinesia in Parkinson's disease. J Neurosci Methods. 2020;

23. Li MH, Mestre TA, Fox SH, Taati B. Vision-based assessment of parkinsonism and levodopa-induced dyskinesia with pose estimation. J Neuroeng Rehabil. 2018; computer vision: Can it measure Parkinson's finger tap bradykinesia? J Neurol Sci. 2020; 
392 25. Shin JH, Ong JN, Kim R, Park S min, Choi J, Kim HJ, et al. Objective measurement of 393 limb bradykinesia using a marker-less tracking algorithm with 2D-video in PD patients. 394 Park Relat Disord. 2020;

395 26. Simon T, Joo H, Matthews I, Sheikh Y. Hand keypoint detection in single images using 396 multiview bootstrapping. In: Proceedings - 30th IEEE Conference on Computer Vision $397 \quad$ and Pattern Recognition, CVPR 2017. 2017.

398 27. Nath T, Mathis A, Chen AC, Patel A, Bethge M, Mathis MW. Using DeepLabCut for 3D 399 markerless pose estimation across species and behaviors. Nat Protoc. 2019;

400 28. Chen K, Gabriel P, Alasfour A, Gong C, Doyle WK, Devinsky O, et al. Patient-specific $401 \quad$ pose estimation in clinical environments. IEEE J Transl Eng Heal Med. 2018;

402 29. Seethapathi N, Wang S, Saluja R, Blohm G, Kording KP. Movement science needs 403 different pose tracking algorithms. arXiv Prepr arXiv190710226. 2019; 


\section{FIGURE CAPTIONS}

407 Figure 1. We used a three-step workflow to measure movement frequency from smartphone

408 videos. Participants first provided a video recording of themselves performing the task of interest

409 (we assessed five different tasks in this study: finger tapping, hand opening/closing, hand

410 pronation/supination, toe tapping, and leg agility or heel tapping). Next, we used OpenPose to

411 estimate body and hand keypoint locations on each video. We then developed post-processing

412 software to calculate movement frequency using the OpenPose outputs.

413 Figure 2. A) Representative example of stillframes from an OpenPose output video of a

414 participant performing the finger tapping task (left) and violin plots showing comparisons of

415 individual participant mean finger tapping frequencies measured manually (“actual”, blue) and

416 using our pose estimation workflow (“OpenPose”, red) across all four target frequencies (right).

417 B) Violin plots showing event time errors (i.e., differences between video timestamps of finger

418 taps as measured manually and using pose estimation) for each individual finger tap across all

419 participants. In Figures $2 \mathrm{~A}$ and 2B, white circles indicate group mean values, shaded circles

420 indicate individual data points. These conventions are consistent across Figures 2-6. C) Scatter

421 plots showing relationships between the video timestamps of all individual finger taps across all

422 participants as measured manually (x-axis) or using our pose estimation workflow (y-axis) across

423 all four target frequencies. Relevant correlation coefficients are included in the Results section of

424 the text for Figures 2-6.

425 Figure 3. A) Representative example of stillframes from an OpenPose output video of a

426 participant performing the hand opening and closing task (left) and violin plots showing

427 comparisons of individual participant mean hand closure frequencies measured manually

428 ("actual”, blue) and using our pose estimation workflow ("OpenPose", red) across all four target 
429 frequencies (right). B) Violin plots showing event time errors (i.e., differences between video

430 timestamps of hand closures as measured manually and using pose estimation) for each

431 individual hand closure across all participants. C) Scatter plots showing relationships between

432 the video timestamps of all individual hand closures across all participants as measured manually

433 (x-axis) or using our pose estimation workflow (y-axis) across all four target frequencies.

434 Figure 4. A) Representative example of stillframes from an OpenPose output video of a 435 participant performing the hand pronation/supination task (left) and violin plots showing 436 comparisons of individual participant mean hand pronation/supination frequencies measured

437 manually (“actual”, blue) and using our pose estimation workflow (“OpenPose”, red) across all

438 four target frequencies (right). B) Violin plots showing event time errors (i.e., differences

439 between video timestamps of hand pronation or supination events as measured manually and

440 using pose estimation) for each individual hand pronation or supination event across all

441 participants. C) Scatter plots showing relationships between the video timestamps of all

442 individual hand pronation or supination events across all participants as measured manually (x-

443 axis) or using our pose estimation workflow (y-axis) across all four target frequencies.

444 Figure 5. A) Representative example of stillframes from an OpenPose output video of a

445 participant performing the toe tapping task (left) and violin plots showing comparisons of

446 individual participant mean toe tapping frequencies measured manually ("actual”, blue) and

447 using our pose estimation workflow (“OpenPose”, red) across all four target frequencies (right).

448 B) Violin plots showing event time errors (i.e., differences between video timestamps of toe taps

449 as measured manually and using pose estimation) for each individual toe tap across all

450 participants. C) Scatter plots showing relationships between the video timestamps of all 
451 individual toe taps across all participants as measured manually (x-axis) or using our pose

452 estimation workflow (y-axis) across all four target frequencies.

453 Figure 6. A) Representative example of stillframes from an OpenPose output video of a

454 participant performing the leg agility (i.e., heel tapping) task (left) and violin plots showing

455 comparisons of individual participant mean heel tapping frequencies measured manually

456 ("actual”, blue) and using our pose estimation workflow ("OpenPose”, red) across all four target

457 frequencies (right). B) Violin plots showing event time errors (i.e., differences between video

458 timestamps of heel taps as measured manually and using pose estimation) for each individual

459 heel tap across all participants. C) Scatter plots showing relationships between the video

460 timestamps of all individual heel taps across all participants as measured manually (x-axis) or

461 using our pose estimation workflow (y-axis) across all four target frequencies. 
video

bioRxiv preprint doi: https://doi.org/10.1101/2021.02.01.429161; this version posted February 2, 2021. The copyright holder for this preprint

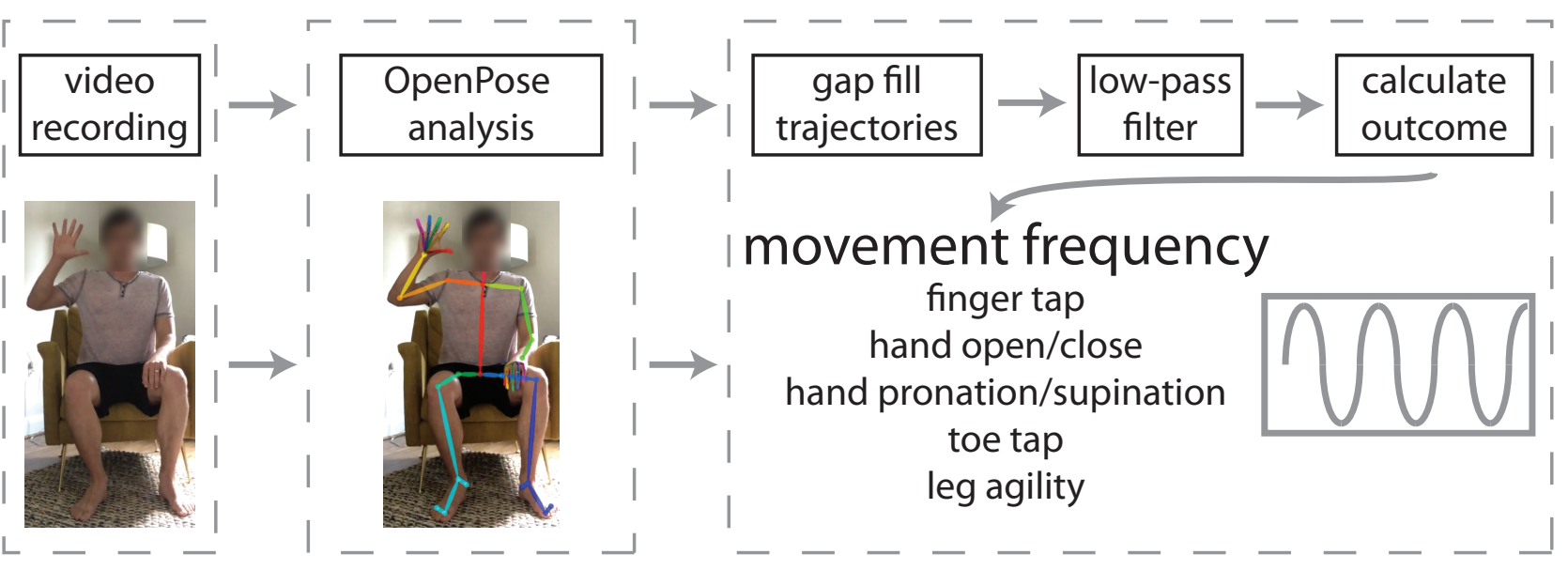

Figure 1. 
A bioRxiv preprint quindes:tgajorg/10.1101/2021.02.01.429161; this version posted February 2, 2021. The coplpight holder for this preprint
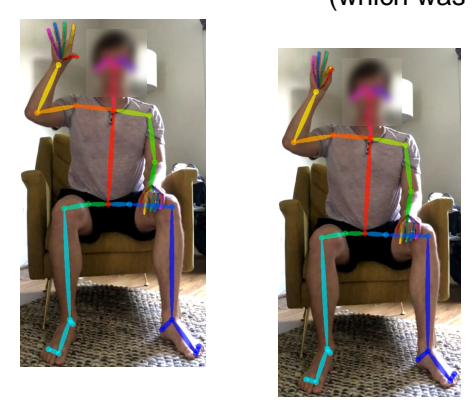

C

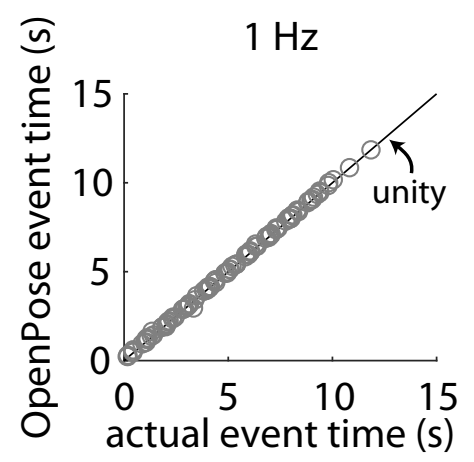

$1 \mathrm{~Hz}$
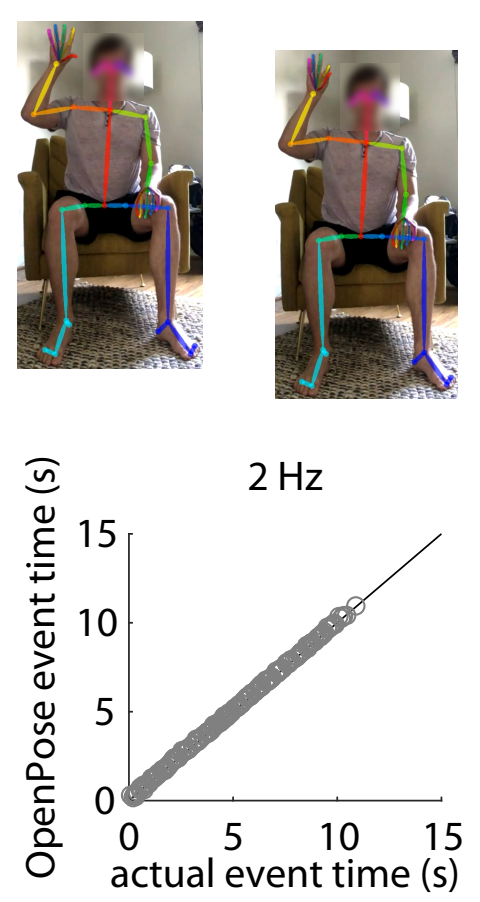
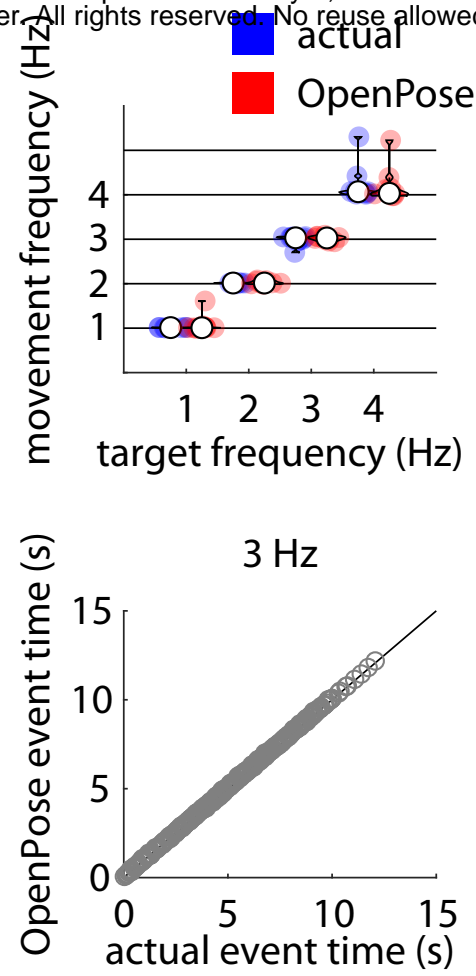
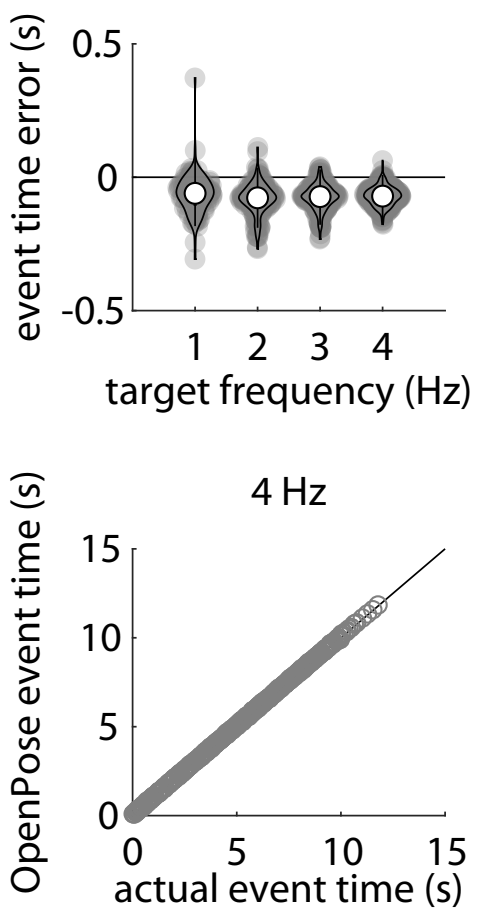

Figure 2. 


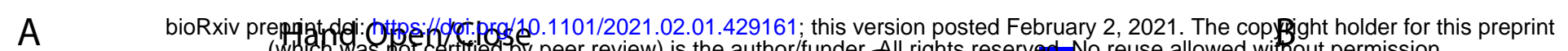
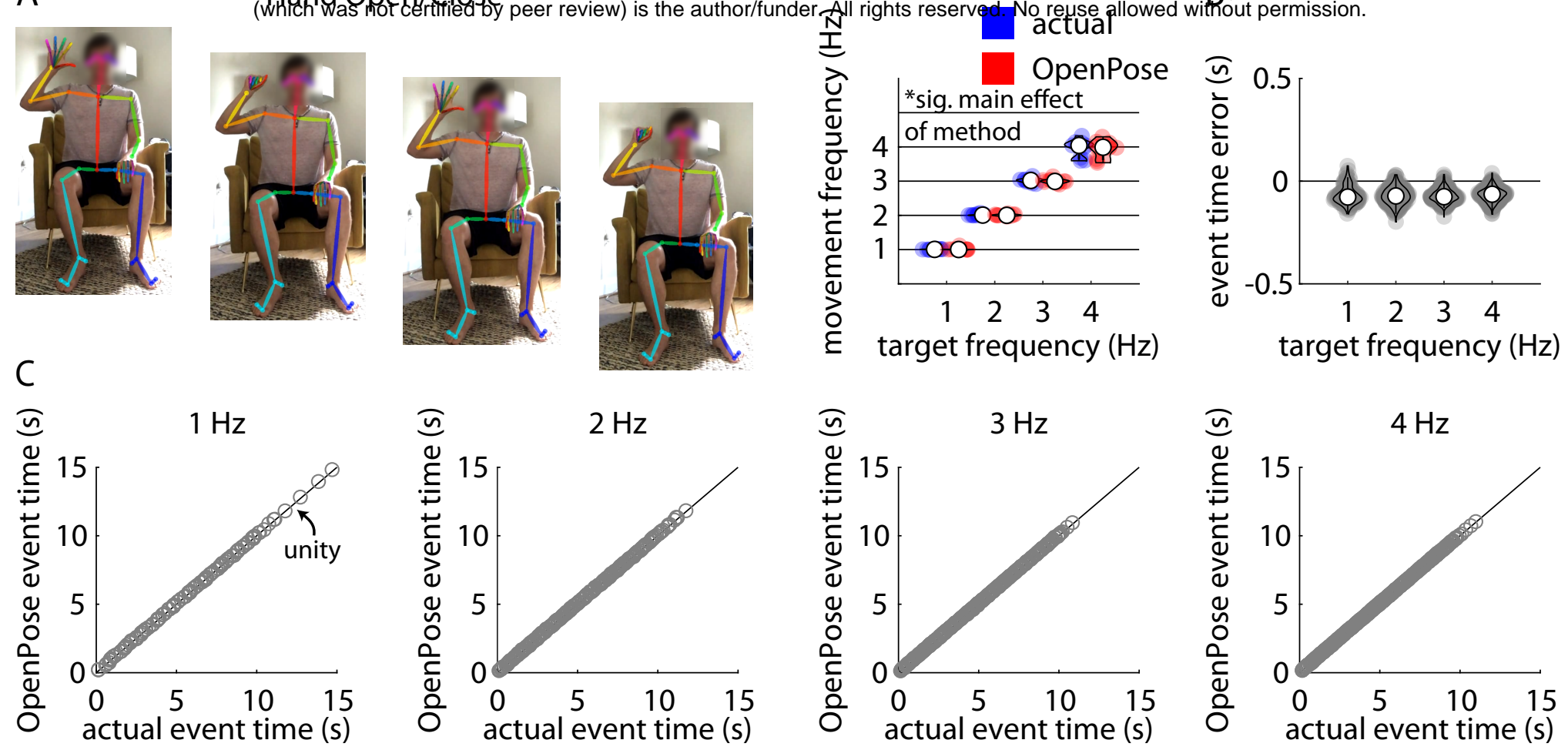

Figure 3. 

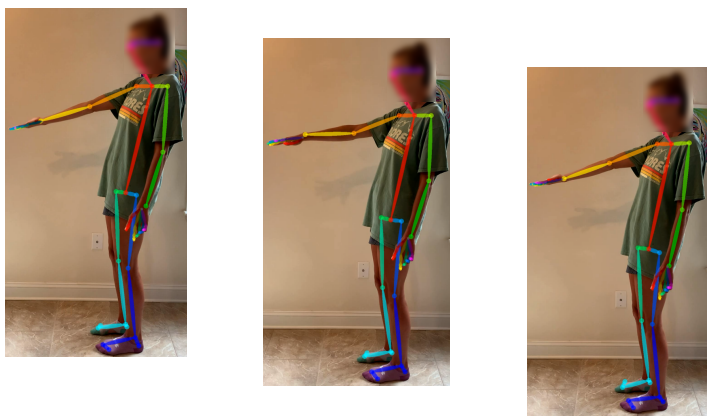

$1 \mathrm{~Hz}$
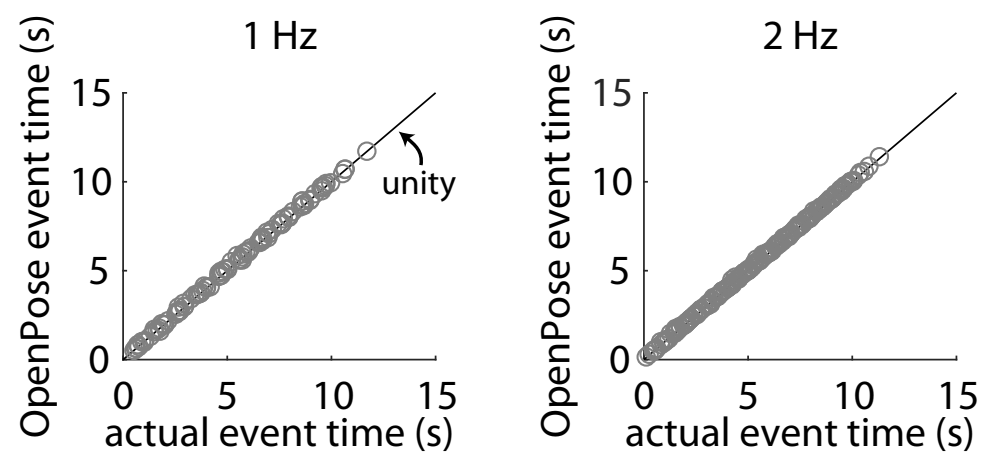
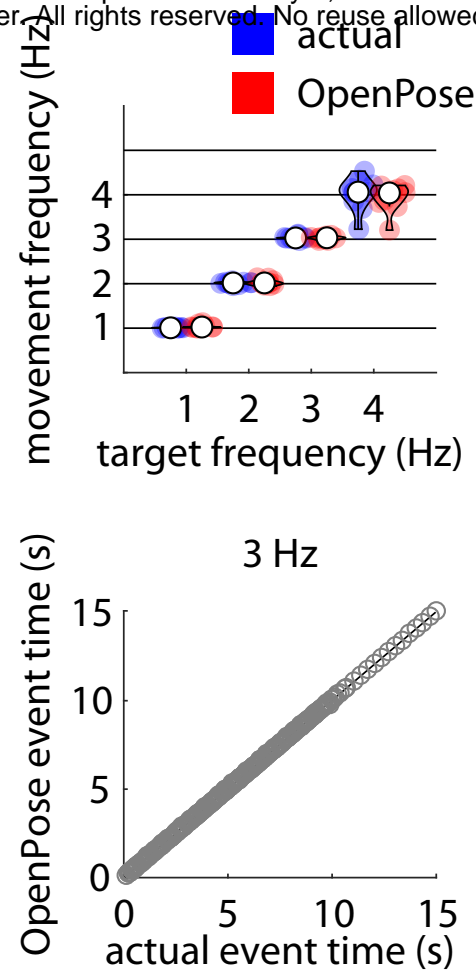
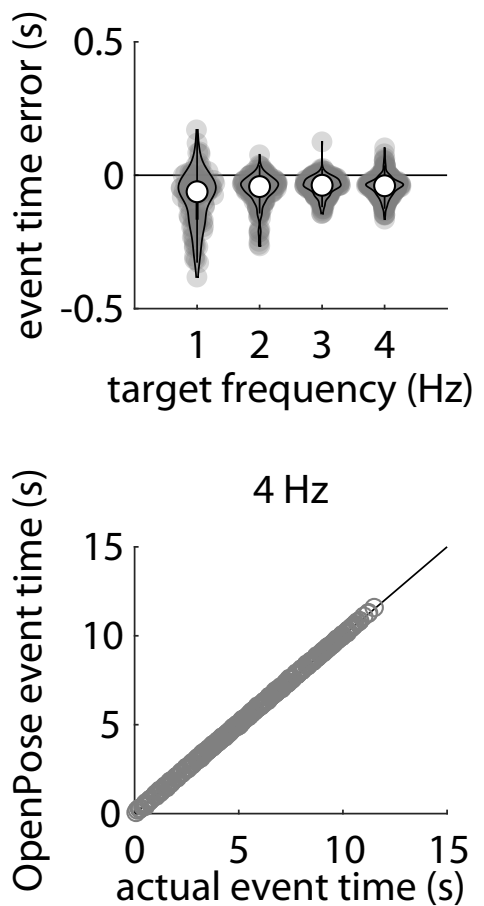

Figure 4 . 
